\title{
The Effects of Interval Running Exercise on Acute Calorie Expenditure
}

\author{
Mehmet Yıldız and Zeki Aky1ldız \\ School of Physical Education and Sports, Afyon Kocatepe University, Afyon 03200, Turkey
}

\begin{abstract}
Interval training is getting more attention due to the rapid improvement in fitness and health level. This is so important for the people who have no much time to spend in gym or sports areas. Therefore, burning much more calories in a short time is getting important for these busy people. The aim of this study was to investigate the effect of interval running exercises on acute calorie consumption. Thirteen female and ten male sedentary collegian students (age: $19.48 \pm 1.64$, height: $171.95 \pm 8.14$, weight: $65.91 \pm$ 14.69) participated in the current study. All participates performed a steady state running for 30 min with $8 \mathrm{~km} /$ hour speed and interval running ( $6 \mathrm{~km} / \mathrm{h}$ and $10 \mathrm{~km} / \mathrm{h}$ with 1 minute interval) for $30 \mathrm{~min}$ on a treadmill on different days. Their calorie expenditure and other related gas parameters were measured with a mobile gas analyzer. The paired t-test was used to compare two protocols for the whole group. Moreover, the Wilcoxon test was used to measure in order to detect significant differences between steady state and interval running protocols for male and female groups. The results showed that there was no significant difference in calorie consumption, $\mathrm{CO}_{2}$ production and RER values, while the only difference was observed in $\mathrm{O}_{2}$ cost $(p<0.01)$ and heart rate in the whole, male and female groups $(p<0.05)$. As a conclusion, it was seen that interval running caused no more calorie expenditure than the classical steady state running acutely. Therefore, calorie burning may be delayed. There is a need for further study in this area.
\end{abstract}

Key words: Interval running, calorie, expenditure.

\section{Introduction}

Excessive calorie intake and a sedentary lifestyle are seen as the main reason of epidemic of metabolic syndrome which is a cluster of cardiovascular risk factors including high blood pressure, dyslipidemia, insulin resistance, and obesity [1]. Almost 300 million people have been affected from these syndromes [2]. However, it is well known that increased physicals activities are the most effective and low-cost treatment for the prevention of such symptoms related with metabolic disorders [3-7]. However, most of the people are reluctant to engage in exercise because of lack of time, despite the obvious therapeutic potential of exercise interventions [8]. The other problem is what type of exercise or machines is chosen for the general health improvement. Because new training methods and machines are continually developed and often touted as being more effective than the

Corresponding author: Mehmet Yıldız, Ph.D., researcher, research fields: physical activity and public health. traditional training methods.

The main goal of all these systems is to burn more calories [9]. Therefore, many studies were conducted to evaluate burning more calories according to newer aerobic devices in traditional forms of aerobic exercise [9-12], and walking methods (modified forms of walking versus regular walking or walking versus running) [13-16].

At this point, interval training is getting more attention for a better health and fitness level. During interval exercises, the body's homeostasis is disrupted and re-established. This makes interval exercises more effective than steady state aerobic exercise because of an increasing metabolic rate following a workout [17]. It is well known that interval training reverses the risk factors of metabolic syndrome [2], decreasing cardiovascular-disease risk factors in obese adolescents [2], increasing proteins that transport fatty acids across the mitochondrial membrane [18] and have a good effect on vascular health [19]. Moreover, muscle oxidative capacity of carbohydrates and fat [20, 
21] and skeletal muscle enzyme activity [22-24] increase more when compared with the traditional continuous aerobic training (same extend). Furthermore, it has been indicated that interval running is seen as more motivating and it is perceived as more enjoyable than other moderate intensity continuous exercises [2]. These results may be so important for the people who want to burn more calories and have no much time to spend in gym or sports areas.

Although it is well known that interval training reverses the risk factors of metabolic syndrome and has a positive effect on health, the acute effect of interval training on calorie conception is unknown. We support the hypothesis that by altering the body's homeostasis during interval exercises, the body may burn more calories to adapt these changes and to overcome exercises intensity. Therefore, the aim of the current study was to investigate effect of interval running on acute calorie consumption.

\section{Material and Methods}

\subsection{Participants}

Thirteen female and ten male sedentary collegian students (age: $19.48 \pm 1.64$, height: $171.95 \pm 8.14$, weight: $65.91 \pm 14.69$ ) were volunteered to participate in the current study. The participants did not have any serious injuries for at least six months. The study was approved by the ethics committee of the Osmangazi University, Turkey, in accordance with the ethical standards of the Helsinki Declaration. A written informed consent form was signed by all participants before the start of the study.

The procedures are as follow. After 10 minutes of warm-up exercise which included cycling on a bicycle ergometer, free stretching, participants familiarized with running on a treadmill. All of the participants performed a classical SS (steady state) running for 30 min with $8 \mathrm{~km} /$ hour speed and a interval running (6 $\mathrm{km} / \mathrm{h}$ and $10 \mathrm{~km} / \mathrm{h}$ with 1 minute interval) for $30 \mathrm{~min}$ on a treadmill with three days interval. Their calorie expenditure and other related gas parameters were tested using the Respiratory Gas Exchange Analyzer VO2000 (Medgraphic, USA).

\subsection{Measuring Calorie Consumption}

Caloric expenditure was determined using the gas analyzer VO2000 (MedGraphics, USA) which was calibrated before and after each test using a $2 \mathrm{~L}$ syringe and gases of the known concentration. Heart rate measurements were carried out using a telemetry device (Polar Oy Electro, Finland). The VO2000 measures oxygen uptake, carbon dioxide output and exhaled air volume in liters per minute. Having these parameters, the software calculated the consumed energy expressed in kilocalories per minute (kcal/min).

\subsection{Statistical Analysis}

Normality of the distribution was calculated with Kolmogorov Smirnov test, and homogeneity of variance was calculated with Levene test. A paired t-test was used to compare two protocols for the whole group. The Wilcoxon test was used to detect whether there were significant differences between steady state and interval running protocols for male and female groups when the data were not normally distributed. All data were expressed as mean and SD (standard deviation), and all analyses were done through SPSS 18.0 (Statistical Package for Social Sciences, Chicago, IL, USA).

\section{Results}

Table 1 shows the means and SDs of calorie expenditure ( $\mathrm{kcal} / 30 \mathrm{~min}), \mathrm{O}_{2}$ consumption $(\mathrm{mL} / \mathrm{min})$, $\mathrm{CO}_{2}$ production $(\mathrm{mL} / \mathrm{min})$, RER and $\mathrm{HR}(\mathrm{bpm} / \mathrm{min}$ ) results. There was not any statistically significant difference in terms of calorie consumption, $\mathrm{CO}_{2}$ production and RER when steady state and interval running protocols were compared in total, male and female groups. When comparing $\mathrm{O}_{2}(\mathrm{~mL} / \mathrm{min})$ used during the exercises, the participants displayed 
Table 1 Comparison of steady state and interval running trials of participants [total $(n=23)$, female $(n=13)$ and male $(n=$ 10)] for calorie burning, $\mathrm{O}_{2}$ consumption, $\mathrm{CO}_{2}$ production, $\mathrm{RER}$ and $\mathrm{HR}$ values.

\begin{tabular}{|c|c|c|c|c|}
\hline Tests & Trials & $\begin{array}{l}\text { Total } \\
\bar{X} \pm \text { sd }\end{array}$ & $\begin{array}{l}\text { Male } \\
\bar{X} \pm \mathrm{sd}\end{array}$ & $\begin{array}{l}\text { Female } \\
\bar{X} \pm \mathrm{sd}\end{array}$ \\
\hline \multirow{2}{*}{ Calorie (kcal/30 min) } & SS & $298.87 \pm 64.66$ & $333.50 \pm 78.09$ & $263.38 \pm 27.83$ \\
\hline & Int & $295.13 \pm 63.41$ & $345.70 \pm 66.45$ & $256.23 \pm 16.97$ \\
\hline \multirow{2}{*}{$\mathrm{O}_{2}(\mathrm{~mL} / \mathrm{min})$} & SS & $1,975.60 \pm 420.86 *$ & $2,257.80 \pm 479.9 *$ & $1,758.53 \pm 185.88^{*}$ \\
\hline & Int & 1,864.26 \pm 398.99 & $2,180.20 \pm 424.41$ & $1,621.23 \pm 97.93$ \\
\hline \multirow{2}{*}{$\mathrm{CO}_{2}(\mathrm{~mL} / \mathrm{min})$} & SS & $1,911.00 \pm 440.50$ & $2,178.80 \pm 518.33$ & $1,675.00 \pm 220.15$ \\
\hline & Int & $1,980.22 \pm 385.28$ & $2,245.90 \pm 397.01$ & $1,699.76 \pm 115.89$ \\
\hline \multirow{2}{*}{ RER } & SS & $0.95 \pm 4.63$ & $0.93 \pm 4.86$ & $0.96 \pm 4.32$ \\
\hline & Int & $0.98 \pm 6.34$ & $0.98 \pm 5.75$ & $0.98 \pm 6.91$ \\
\hline \multirow{2}{*}{ HR (bpm/min) } & SS & $165.30 \pm 15.79 *$ & $157.80 \pm 11.18^{*}$ & $171.07 \pm 16.73^{*}$ \\
\hline & Int & $156.57 \pm 13.05$ & $148.20 \pm 10.08$ & $163.00 \pm 11.51$ \\
\hline
\end{tabular}

${ }^{*} p<0.05$, SS: Steady state, Int: Interval, RER: Respiratory Exchange Ratio.

significantly $\left(p \leq 0.05\right.$ ) greater $\mathrm{O}_{2}$ consumption in steady state running (total group: 1,975.60 \pm 420.86 $\mathrm{mL} / \mathrm{min}$; male: $2,257.80 \pm 479.9 \mathrm{~mL} / \mathrm{min}$ and female: $1,758.53 \pm 185.88 \mathrm{~mL} / \mathrm{min}$ separately) compared with interval running protocol (total: 1,864.26 \pm 398,99 $\mathrm{mL} / \mathrm{min}$; male: 2,180.20 $\pm 424.41 \mathrm{~mL} / \mathrm{min}$; female: $1,621.23 \pm 97.93 \mathrm{~mL} / \mathrm{min}$ separately) in whole, male and female groups. Similarly, the HR was significantly $(p<0.05)$ higher during steady state running (total: $165.30 \pm 15.79$ bpm; male: $157.80 \pm$ $11.18 \mathrm{bpm}$; female: $171.07 \pm 16.73$ separately) when compared to interval running (total: $156.57 \pm 13.05$ bpm; male: $148.20 \pm 10.08$ bpm, female: $163.00 \pm$ 11.51 bpm, separately) in total, male and female groups.

\section{Discussion}

Revealing the actual energy expenditure ratio of the exercise activity is so important for the people who want to burn more calories and have no much time to spend in gym or sports areas. During interval exercises, the body's homeostasis is disrupted and re-established. This makes interval exercises more effective than continuous aerobic exercise because of increasing metabolic rate following a workout. Although it is well known that interval training reverses the risk factors of metabolic syndrome and has a positive effect on health, the acute effect of interval training on calorie expenditure is unknown. This current study has been the first one to investigate the effect of interval running on acute calorie consumption.

Our findings showed that there was no significant difference for the total caloric expenditure when steady state and interval running protocols were compared. A hypothesis explaining these findings could be that interval running was separated as high and low intensity workout with one-minute intervals. Therefore, one-minute intervals might not be enough to reach steady state level and also to recover properly for performing another high-intensity interval during running. This might cause an increase in EPOC (excessive post-exercise oxygen consumption) which means the elevation in $\mathrm{O}_{2}$ consumption above the resting level after exercise [25]. EPOC is initially thought to contribute to the energy cost of exercise [17]. Several factors contribute to the EPOC. First, some of the $\mathrm{O}_{2}$ consumed early in the recovery period is used to re-synthesize store PC in the muscle and replace $\mathrm{O}_{2}$ stores in both muscle and blood. Other factors that contribute to the "slow" portion of the EPOC include an elevated blood temperature, hormones and $\mathrm{HR}, \mathrm{O}_{2}$ required to convert lactic acid to glucose (gluconeogenesis) [26]. Supporting this hypothesis, Ref. [17] compared the EPOC after a sub-maximal continuous running and a supra-maximal 
interval running. They found a higher post-exercise metabolic rate following an interval running $(20 \times$ 1-min at $105 \% \mathrm{VO}_{2}$ max with 2-min rest) than they did after steady state running (30-min at $70 \% \mathrm{VO}_{2}$ max). Similarly, Ref. [27] investigated the effects of steady state and interval exercise on energy expenditure and substrate oxidation over a 24-h period. They found that more calories were burnt during the 24 hours following an interval workout $(15 \times 2$-min at $100 \% \mathrm{CO}_{2}$ max with 2-min rest periods) than the period after continuous exercise (60-min at $50 \% \mathrm{CO}_{2}$ max). Moreover, Ref. [28] compared an endurance exercise with an HIIT (high intensity interval training). Although they found significant lower energy cost at the end of the study in HIIT workout, a 9-fold greater reduction in skinfold thickness was observed in the HIIT group. Probably, HIIT heightens the of excessive post-exercise oxygen consumption known as "after burn" [29], and this may increase up regulation of enzymes responsible for beta-oxidation and the body's potential to use lipids as an energy substrate [28].

Another finding in our study was that when comparing $\mathrm{O}_{2}(\mathrm{~mL} / \mathrm{min})$ used during the exercises, the participants displayed significantly greater $\mathrm{O}_{2}$ consumption in steady state running. Moreover, average HR was lower during the interval running than steady state running. It is well known that in the transition from rest to light or moderate exercise, $\mathrm{O}_{2}$ consumption increases rapidly and reaches steady state within one to four minutes [30]. The energy to perform long-term exercise (i.e., more than ten minutes) comes primarily from aerobic metabolism. A steady-state oxygen uptake can generally be maintained during sub-maximal exercise of moderate duration [26]. Moreover, the time to reach steady state is shorter in trained subjects than in untrained subjects [31]. This difference in the time course of oxygen uptake at the one set of exercise between trained and untrained subjects results in the trained subjects having a lower oxygen deficit when compared to the untrained. Furthermore, it is generally believed that most of the ATP (adenosine triphosphate) production used to provide energy muscular contraction during the early stages of an incremental exercise comes from aerobic sources [26]. However, as the exercise intensity increases, blood levels of lactic acid begin to rise in an exponential fashion. This appears in untrained subjects around $50 \%$ to $60 \%$ of $\mathrm{CO}_{2}$ max. While it occurs at higher work rates in trained subjects (i.e., $65 \%-80 \% \mathrm{CO}_{2} \max$ ) [32]. Therefore, we think that during interval running the participants had more oxygen deficit, because all participants in our study were untrained. In additionally, it has been demonstrated that heart rate begins to increase within the first second after muscular contraction begins [33]. If the work rate is constant and below the lactate threshold, a steady-state plateau in heart rate is reached within two to three minutes. However, heart rates decrease rapidly in the first minute of recovery. Therefore, it is inevitable that the heart rate is lower during in interval running when considering that interval running was separated as high and low intensity workout with one-minute intervals.

Consequently, calorie expenditure in interval running was not significantly different when it was compared with steady state running. This might cause an increase in EPOC. Therefore, calorie burning may be delayed. There is a need for further study in this area.

\section{Acknowledgments}

This study was presented as a verbal statement at the 14th International Sports Sciences Congress that was held in Antalya, TURKEY.

\section{References}

[1] Hawley, J. A., and Gibala, M. J. 2009. "Exercise Intensity and Insulin Sensitivity: How Low Can You Go?” Diabetologia 52 (9): 1709-13.

[2] Tjønna, A. E., Lee, S. J., Rognmo, Ø., Stølen, T. O., Bye, A., Haram, P. M., and Kemi, O. J. 2008. "Aerobic Interval Training versus Continuous Moderate Exercise as a Treatment for the Metabolic Syndrome.” Circulation 118 (4): 346-54. 
[3] Goodyear, L. J., and Kahn B. B. 1998 "Exercise, Glucose Transport, and Insulin Sensitivity.” Annu Rev. Med. 49: 235-61.

[4] Hawley, J. A. 2004. "Exercise as a Therapeutic Intervention for the Prevention and Treatment of Insulin Resistance.” Diabetes/Metabolism Research and Reviews 20 (5): 383-93.

[5] Hawley, J. A., and Lessard, S. J. 2008. "Exercise Training - Induced Improvements in Insulinaction.” Acta Physiologica 192 (1): 127-35.

[6] Pedersen, B. K., and Saltin, B. 2006. "Evidence for Prescribing Exercise as Therapy in Chronic Disease.” Scandinavian Journal of Medicine \& Science in Sports 16 (S1): 3-63.

[7] Seals, D. R., Hagberg, J. M., Hurley, B. F., Ehsani, A. A., amd Holloszy, J. O. 1984. "Effects of Endurance Training on Glucose Tolerance and Plasma Lipid Levels in Older Men and Women.” Jama 252 (5): 645-9.

[8] Bartlett, J. D., Close, G. L., MacLaren, D. P., Gregson, W., Drust, B., and Morton, J. P. 2011. "High-Intensity Interval Running Is Perceived to Be More Enjoyable Than Moderate-Intensity Continuous Exercise: Implications for Exercise Adherence.” Journal of Sports Sciences 29 (6): 547-53.

[9] Falcone, P. H., Tai, C. Y., Carson, L. R., Joy, J. M., Mosman, M. M., McCann, T. R., and Moon, J. R. 2015. "Caloric Expenditure of Aerobic, Resistance, or Combined High-Intensity Interval Training Using a Hydraulic Resistance System in Healthy Men." The Journal of Strength \& Conditioning Research 29 (3): 779-85.

[10] Kravitz, L., Robergs, R. A., Heyward, V. H., Wagner, D. R., and Powers, K. 1997. "Exercise Mode and Gender Comparisons of Energy Expenditure at Self-selected Intensities." Medicine and Science in Sports and Exercise 29 (8): 1028-35.

[11] Thomas, T. R., Feiock, C. W., and Araujo, J. 1989. "Metabolic Responses Associated with Four Modes of Prolonged Exercise.” The Journal of Sports Medicine and Physical Fitness 29 (1): 77-82.

[12] Zeni, A. I., Hoffman, M. D., and Clifford, P. S. 1996. "Energy Expenditure with Indoor Exercise Machines." Jama 275 (18): 1424-7.

[13] Jeukendrup, A. E., Thielen, J. J., Wagenmakers, A. J., Brouns, F., and Saris, W. H. 1998. "Effect of Medium-Chain Triacylglycerol and Carbohydrate Ingestion during Exercise on Substrate Utilization and Subsequent Cycling Performance." The American Journal of Clinical Nutrition 67 (3): 397-404.

[14] Porcari, J. P., Hendrickson, T. L., Walter, P. R., Terry, L., and Walsko, G. 1997. "The Physiological Responses to Walking with and without Power Poles ${ }^{\mathrm{TM}}$ on Treadmill
Exercise.” Research Quarterly for Exercise and Sport 68 (2): 161-6.

[15] Rodgers, C. D., VanHeest, J. L., and Schachter, C. L. 1995. "Energy Expenditure during Submaximal Walking with Exerstriders." Medicine and Science in Sports and Exercise 27.

[16] Hall, C., Figueroa, A., Fernhall, B. O., and Kanaley, J. A. 2004. "Energy Expenditure of Walking and Running: Comparison with Prediction Equations.” Medicine and Science in Sports and Exercise 36: 2128-34.

[17] Laforgia, J., Withers, R. T., Shipp, N. J., and Gore, C. J. 1997. "Comparison of Energy Expenditure Elevations after Submaximal and Supramaximal Running.” Journal of Applied Physiology 82 (2): 661-6.

[18] Talanian, J. L., Holloway, G. P., Snook, L. A., Heigenhauser, G. J., Bonen, A., and Spriet, L. L. 2010. "Exercise Training Increases Sarcolemmal and Mitochondrial Fatty Acid Transport Proteins in Human Skeletal Muscle." American Journal of Physiology-Endocrinology and Metabolism 299 (2): E180-8.

[19] Rakobowchuk, M., Tanguay, S., Burgomaster, K. A., Howarth, K. R., Gibala, M. J., and MacDonald, M. J. 2008. "Sprint Interval and Traditional Endurance Training Induce Similar Improvements in Peripheral Arterial Stiffness and Flow-Mediated Dilation in Healthy Humans.” American Journal of Physiology-Regulatory, Integrative and Comparative Physiology 295 (1): R236-42.

[20] Burgomaster, K. A., Howarth, K. R., Phillips, S. M., Rakobowchuk, M., MacDonald, M. J., McGee, S. L., and Gibala, M. J. 2008. "Similar Metabolic Adaptations during Exercise after Low Volume Sprint Interval and Traditional Endurance Training in Humans.” The Journal of Physiology 586 (1): 151-60.

[21] Chilibeck, P. D., Bell, G. J., Farrar, R. P., and Martin, T. P. 1998. "Higher Mitochondrial Fatty Acid Oxidation following Intermittent Versus Continuous Endurance Exercise Eraining." Canadian Journal of Physiology and Pharmacology 76 (9): 891-4.

[22] Gibala, M. J., Little, J. P., Van Essen, M., Wilkin, G. P., Burgomaster, K. A., Safdar, A., and Tarnopolsky, M. A. 2006. "Short-Term Sprint Interval versus Traditional Endurance Training: Similar Initial Adaptations in Human Skeletal Muscle and Exercise Performance.” The Journal of Physiology 575 (3): 901-11.

[23] MacDougall, J. D., Hicks, A. L., MacDonald, J. R., McKelvie, R. S., Green, H. J., and Smith, K. M. 1998. "Muscle Performance and Enzymatic Adaptations to Sprint Interval Training.” Journal of Applied Physiology 84 (6): 2138-42.

[24] Talanian, J. L., Galloway, S. D., Heigenhauser, G. J., 
Bonen, A., and Spriet, L. L. 2007. "Two Weeks of High-Intensity Aerobic Interval Training Increases the Capacity for Fat Oxidation during Exercise in Women.” Journal of Applied Physiology 102 (4): 1439-47.

[25] Gaesser, G. A., and BROOKS, C. A. 1984. "Metabolic Bases of Excess Post-exercise Oxygen." Medicine and Science in Sports and Exercise 16 (1): 29-43.

[26] Powers, C. K., and Howley. E. T. 2011. Exercise Physiology (Theory and Application to Fitness and Performance). New York: McGraw-Hill International edition.

[27] Treuth, M. S., Hunter, G. R., and Williams, M. A. R. T. H. A. 1996. "Effects of Exercise Intensity on 24-h Energy Expenditure and Substrate Oxidation." Medicine and Science in Sports and Exercise 28 (9): 1138-43.

[28] Tremblay, A., Simoneau, J. A., and Bouchard, C. 1994. "Impact of Exercise Intensity on Body Fatness and Skeletal Muscle Metabolism.” Metabolism 43 (7): 814-8.

[29] Bahr, R., and Sejersted, O. M. 1991. "Effect of Intensity of Exercise on Excess Post-Exercise $\mathrm{O}_{2}$ Consumption.” Metabolism 40 (8): 836-41.

[30] Boutellier, U., Giezendanner, D., Cerretelli, P., and DiPrampero, P. E. 1984 "After Effects of Chronic Hypoxia on Effects of Specific Muscle Training on Response and Early Blood Lactatekinetics and on $\mathrm{O}_{2}$ Deficit and Debt." European Journal of Applied Physiology 53 (2): 87-91.

[31] Saltin, B., and Gollnick, P. D. 1988 "Fuel for Muscular Exercise: Role of Carbohydrate. Exercise, nutrition and Energy Metabolism.” İn Exercise, Nutrition and Energy Metabolism, edited by Horton, E., and Terjung, R., 45-71. New York: Macmillan.

[32] Gollnick, P. D., Bayly, W. M., and Hodgson, D. R. 1986. "Exercise Intensity, Training, Diet, and Lactate Concentration in Muscle and Blood.” Medicine \& Science in Sports \& Exercise 18 (3): 334-40.

[33] Maughan, R. J. 1991. "Fluid and Electrolyte Loss and Replacement in Exercise.” Journal of Sports Sciences 9 (S1): 117-42. 